\title{
Estereologia das glândulas bulbouretrais do coelho (Oryctolagus cuniculus) e da cobaia (Cavia porcellus) ${ }^{1}$
}

\author{
Bélgica Vásquez ${ }^{2,3 *}$ e Mariano del Sol ${ }^{4,5}$
}

\begin{abstract}
Vásquez B. \& del Sol M. 2014. [Stereology of the bulbourethral gland of the rabbit (Oryctolagus cuniculus) and guinea pig (Cavia porcellus).] Estereologia das glândulas bulbouretrais do coelho (Oryctolagus cuniculus) e cobaia (Cavia porcellus). Pesquisa Veterinária Brasileira 34(12):1247-1250. Universidad de La Frontera, Avenida Francisco Salazar 01145, Casilla 54-D, Temuco, Chile. E-mail: belgica.vasquez@ufrontera.cl

The bulbourethral glands (GBU) in the rabbit (Oryctolagus cuniculus) and guinea pig (Cavia porcellus) play an important role in reproductive physiology. However, histological and stereological aspects are scarce. Thus, the objective of this research was to compare stereological characteristics between the rabbit and guinea pig GBU as a first approximation in the understanding of morphometric variables involved in reproductive processes. Five rabbits were used and five adult male guinea pigs, healthy, obtained from the Vivarium of the Universidad de La Frontera, Temuco, Chile. Pelvic region was dissected, isolating the GBU of each animal. Was determined weight and volume of each gland. These were fixed in buffered formalin for 24 hours and embedded in paraplast. Serial sections of 4 microns thick, were stained with HE, for stereological analysis. The average glandular cells in the rabbit's GBU was $19.50 \times 10^{5} \mathrm{~mm}^{3}$ (SD 2.35), and for the guinea pig $10.57 \times 10^{5} \mathrm{~mm}^{3}$ (SD 2.07 ), and the average percentage of glandular tissue was $25.52 \%$ (SD 2.20) and $17.20 \%$ (SD 3.33) respectively. All stereological parameters were compared statistically significant difference $(p<0.0001)$. These differences could be explained because there is a closer epithelial cell secretory acinar, smaller lumen diameter and nucleus to cytoplasm ratio in the rabbit's GBU. Thus, the acini of the GBU had a greater number of cells per $\mathrm{mm}^{3}$ in the rabbit's GBU. These parameters can be influenced by hormonal factors, age, seasonal and environmental among others. Consider the morphological characteristics of the GBU in these animals could affect the successful reproduction by the male.
\end{abstract}

INDEX TERMS: Bulbourethral gland, rabbit, guinea pig, stereology.

RESUMO.- As glândulas bulbouretrais (GBU) no coelho (Oryctolagus cuniculus) e na cobaia (Cavia porcellus) desempenham um papel importante na fisiologia reprodutiva. No entanto, seus aspectos histológico e estereológico são escassos. Assim, o objetivo desta pesquisa foi comparar características estereológicas entre as GBU do coelho e da cobaia como um primeiro passo para a compreensão das variáveis

\footnotetext{
${ }^{1}$ Recebido em 22 de setembro de 2013.

Aceito para publicação em 16 de julho de 2014.

${ }^{2}$ Universidad de Tarapacá, General Velásquez 1775, Arica, Chile.

${ }^{3}$ Doutorado en Ciências Morfológicas, Universidad de La Frontera, Temuco, Av. Francisco Salazar 1145, Casilla 54-D, Temuco, Chile. *Autor para correspondência: belgica.vasquez@ufrontera.cl

${ }^{4}$ Faculdade de Medicina, Universidad de La Frontera, Av. Francisco Salazar 1145, Casilla 54-D, Temuco, Chile.E-mail: mariano.delsol@ufrontera.cl

${ }^{5}$ Centro de Investigacão em Ciências Biomédicas, Universidad Autónoma de Chile, Av. Alemania 1090, Temuco, Chile.
}

morfométricas que participam nos processos reprodutivos. Foram utilizados 5 coelhos e 5 cobaias adultos machos, saudáveis, obtidos do Biotério da Universidade de La Frontera, Temuco, Chile. A região pélvica foi dissecada isolando-se a GBU de cada animal. Determinou-se o peso e o volume de cada glândula. Estas foram fixadas em formalina tamponada durante 24 horas e incluídas em paraplast. Cortes seriados de $4 \mu \mathrm{m}$ de espessura foram corados com HE para análise estereológica. A média de células glandulares na GBU do coelho foi $19,50 \times 10^{5} \mathrm{~mm}^{3}$ (DP 2,35) e da cobaia $10,57 \times 10^{5} \mathrm{~mm}^{3}$ (DP 2,07) e a porcentagem média de tecido glandular foi de $25,52 \%$ (DP 2,20) e 17,20\% (DP 3,33), respectivamente. Todos os parâmetros estereológicos comparados tiveram uma diferença estatisticamente significativa $(p<0,0001)$. Estas diferenças poderiam ser explicadas porque há maior proximidade celular do epitélio secretor, menor diâmetro do lúmen dos ácinos e da relação núcleo citoplasma na GBU 
do coelho. Assim, os ácinos da GBU apresentam maior quantidade de células por $\mathrm{mm}^{3}$ do que na GBU do coelho. Estes parâmetros podem ser influenciados por fatores hormonais, etários, sazonais e ambientais, entre outros. Considerar as características morfológicas da GBU nesses animais poderia condicionar o êxito da reprodução por parte do macho.

TERMOS DE INDEXAÇÃO: Glândula bulbouretral, coelho, cobaia, estereologia.

\section{INTRODUÇÃO}

A presença, o número e as características morfológicas da glândula bulbouretral (GBU) nos mamíferos varia de acordo com a espécie. Nos roedores, como a capivara (Hydrochoerus hydrochaeris), não está presente (Fernandez et al. 2010), em coelhos (Oryctolagus cuniculus) é única e está em posição dorsal à uretra e caudal à próstata (Holtz \& Foote 1978, Vásquez \& del Sol 2001, Dimitrov et al. 2012). Em cobaias (Cavia porcellus) a GBU tem uma localização similar a do coelho, no entanto é par (Schilling 1984, Tambrallo \& Fish 2000, Martín et al. 2004, Vásquez \& del Sol 2010). Em espécies como marsupiais podem ser observados três pares de glândulas (De Barros et al. 2013).

0 tecido glandular da GBU do coelho encontra-se constituído por um epitélio simples que varia de cúbico a colunar, subdividido por delgadas trabéculas de tecido conectivo (Holtz \& Foote 1978, Vásquez \& del Sol 2001). Em relação à cobaia, a literatura descreve principalmente os aspectos anatômicos básicos da glândula, enfocando as pesquisas morfológicas com mais detalhes em outras glândulas acessórias, como a próstata e as glândulas vesiculares (Cooper \& Schiller 1975, Cordeiro et al. 2004, Martín et al. 2004, Vásquez \& del Sol 2010).

No homem sua função não está completamente descrita. Está relacionada com a produção de um líquido claro e viscoso, de $\mathrm{pH}$ alcalino que lubrifica a uretra e a glande, neutraliza a acidez da uretra e a secreção vaginal ácida da mulher (Orts 1967, Ninomiya et al. 1995). No sêmen participa também como um fator necessário na coagulação e de defesa urogenital pela secreção de glicoproteínas, tais como o antígeno prostático específico (Pedron et al. 1997, McLellan et al. 2004, Chughtai et al. 2005). Em outros mamíferos sua função é pouco conhecida. No coelho, participa na produção de grânulos de glicogênio, mucosubstâncias neutras e ácidas, sulfatadas e não sulfatadas tanto nas células como em seu produto de secreção (Vásquez \& del Sol 2001). Na cobaia, estudos demonstram que a secreção da GBU apresenta glicoproteínas e frutose (Freund 1969, Moré 1991).

0 estudo da morfologia do sistema reprodutor e de suas características reprodutivas podem ser relevantes para realizar um melhor manuseio destes animais, aumentando a produção e a rentabilidade na sua reprodução. Este fato, adquire importância na cobaia, já que ela faz parte da dieta habitual de populações latinoamericanas (Chauca 1995).

Além disso, o coelho e a cobaia são usados frequentemente como modelos experimentais na área reprodutiva, biomédica e genética. A literatura não relata detalhes sobre descrições morfológicas ou estudos estereológicos da GBU destes animais nem faz comparações que permitam esclarecer sua função. Em vista disso, o objetivo desta pesquisa foi comparar aspectos estereológicos entre as GBU do coelho (Oryctolagus cuniculus) e da cobaia (Cavia porcellus) como primeiro passo para compreender seu papel na reprodução.

\section{MATERIAL E MÉTODOS}

Foram utilizados 5 coelhos (Oryctolagus cuniculus) e 5 cobaias (Cavia porcellus) adultos machos, saudáveis, obtidos do Biotério da Universidad de La Frontera, Temuco, Chile. Os animais foram eutanasiados para extrair em bloco as glândulas acessórias do aparelho genital de ambas as espécies, as quais foram dissecadas mesoscópicamente sob lupa 10x.

As GBU de cada espécie foram separadas e pesadas. 0 volume foi determinado através do método de Scherle (1970). As amostras foram fixadas em formalina tamponada a $10 \%$ e incluídas em Paraplast ${ }^{\circledR}$. Cada amostra foi incluída completa, por seleção aleatória. Foram realizados cortes seriados de $4 \mu \mathrm{m}$ de espessura, utilizando micrótomo rotativo (Microm HM 335 E). Em cada glândula foram realizados 5 cortes para em seguida serem corados com HE. Foram observados 5 campos de cada corte, num total de 125. As lâminas foram analisadas em microscópio óptico Olympus ${ }^{\circledR}$ modelo CX31, com câmara marca Moticam ${ }^{\circledR}$, modelo 480. As imagens foram projetadas num monitor de tela plana, marca Sony ${ }^{\circledR}$. Para realizar a estereologia utilizou-se o teste multipropósito M42.

Os parâmetros estereológicos medidos foram: densidade de número (Nv), densidade de volume (Vv) e número total de células glandulares. Para a análise estatística foi utilizado o programa Stata versão 11.0 e aplicado o teste ANOVA, test t para variâncias diferentes e o teste não paramétrico de Kruskal-Wallis, empregando o mais adequado em cada caso. Foi utilizado um nível de significância de 5\% para os testes estatísticos.

\section{RESULTADOS}

O coelho apresentou uma GBU e a cobaia duas. Em ambas as espécies, a GBU ocupa uma situação dorsal à uretra e caudal à próstata. 0 valor médio do peso da GBU do coelho e da cobaia foi $0,61 \mathrm{~g}$ e $0,23 \mathrm{~g}$, respectivamente e o valor médio do volume foi $0,54 \mathrm{~mm}^{3}$ e $0,16 \mathrm{~mm}^{3}$, respectivamente.

A Nv média das células glandulares da GBU do coelho foi $19,50 \times 10^{5} \mathrm{~mm}^{3}$ (DP 2,35) e a Nv média das células glandulares da GBU da cobaia foi $10,57 \times 10^{5} \mathrm{~mm}^{3}$ (DP 2,07). Estas diferenças foram estatisticamente significativas $(\mathrm{p}<0,0001) .0$ Quadro 1 mostra a análise estatística da Nv da GBU de ambas as espécies.

\begin{tabular}{|c|c|c|c|c|c|c|c|c|}
\hline $\mathrm{Nv}$ & $\begin{array}{c}\text { Média } \\
\text { (células } / \mathrm{mm}^{3} \text { ) }\end{array}$ & D.P. & $\begin{array}{c}\text { Mínimo } \\
\text { (células } / \mathrm{mm}^{3} \text { ) }\end{array}$ & $\begin{array}{c}\text { Máximo } \\
\text { (células } / \mathrm{mm}^{3} \text { ) }\end{array}$ & $\begin{array}{c}\text { E.E. } \\
\text { (células } / \mathrm{mm}^{3} \text { ) }\end{array}$ & $\begin{array}{l}\text { C.V. } \\
(\%)\end{array}$ & $\begin{array}{l}\text { C.E. } \\
(\%)\end{array}$ & $\begin{array}{l}\text { Valor } \\
\text { de p }\end{array}$ \\
\hline GBU Coelho & $19,50 \times 10^{5}$ & 2,35 & $17,19 \times 10^{5}$ & $22,54 \times 10^{5}$ & $1,05 \times 10^{5}$ & 12,03 & 5,38 & $0,79^{*}$ \\
\hline GBU Cobaia & $10,57 \times 10^{5}$ & 2,07 & $8,30 \times 10^{5}$ & $13,12 \times 10^{5}$ & $0,92 \times 10^{5}$ & 19,5 & 8,72 & $0,64^{* *}$ \\
\hline
\end{tabular}


A Vv média do tecido glandular da GBU do coelho e da cobaia foi de $25,52 \%$ (DP 2,20) e $17,20 \%$ (DP 3,33), respectivamente. 0 valor de p que compara a porcentagem de tecido glandular em ambas as estruturas foi 0,0001. 0 Quadro 2 mostra a análise estatística da Vv da GBU de ambas as espécies.

O número total de células glandulares do coelho foi em média $10,53 \times 10^{5}$ e da cobaia foi em média $1,69 \times 10^{5}$.

Quadro 2. Média da densidade de volume e parâmetros estatísticos do tecido glandular da GBU do coelho (Orytolagus coniculus) e da cobaia (Cavia porcellus)

\begin{tabular}{ccccccccc}
\hline Vv & $\begin{array}{c}\text { Média } \\
\%\end{array}$ & D.P. & $\begin{array}{c}\text { Mínimo } \\
\%\end{array}$ & $\begin{array}{c}\text { Máximo } \\
\%\end{array}$ & $\begin{array}{c}\text { E.E. } \\
\%\end{array}$ & $\begin{array}{c}\text { C.V. } \\
(\%)\end{array}$ & $\begin{array}{c}\text { C.E. } \\
(\%)\end{array}$ & $\begin{array}{c}\text { Valor } \\
\text { de p }\end{array}$ \\
\hline GBU Coelho & 25,52 & 2,20 & 23,60 & 28,13 & 0,98 & 8,01 & 3,85 & 0,8884 \\
GBU Cobaia & 17,20 & 3,33 & 13,54 & 21,47 & 1,49 & 19,35 & 8,66 & 0,96
\end{tabular}

Teste ANOVA.

\section{DISCUSSÃO}

Na literatura não foi possível encontrar estudos estereológicos da GBU do coelho (Oryctolagus cuniculus) e da cobaia (Cavia porcellus). No entanto, existem pesquisas em outros animais, como na viscacha (Lagostomus maximus maximus) (Chaves et al. 2011) e na cutia (Dasyprocta prymnolopha) (Ayres de Menezes et al. 2010), na qual são descritas características morfológicas macroscópicas e histológicas similares à GBU da cobaia.

Através deste estudo, demonstrou-se que a GBU do coelho tem em média um maior número de células glandulares por $\mathrm{mm}^{3}$ do que a cobaia, diferença estatisticamente significativa $(\mathrm{p} \leq 0,05)$. Isto poderia ser explicado porque as células glandulares do epitélio secretor túbulo-acinoso do coelho estão mais próximas entre si, o lúmen dos acinos é de menor diâmetro e a relação núcleo citoplasma é menor comparada com os ácinos da GBU da cobaia (Holtz \& Foote 1978, Vásquez \& del Sol 2001), consequentemente, os ácinos apresentam maior quantidade de células por $\mathrm{mm}^{3}$.

Em mamíferos machos, as glândulas acessórias diferenciam-se de outros órgãos do corpo pelo caráter altamente especializado de suas secreções, assim como por sua dependência ao estímulo androgênico para o desenvolvimento e atividade secretora (Mann 1974). Diversos autores relatam que os andrógenos geram mudanças morfológicas e ultraestruturais no tecido das glândulas acessórias do sistema reprodutor destes animais (Barnes 1972, Tilley et al. 1989, Cordeiro et al. 2004). Em nosso estudo a porcentagem de tecido glandular no coelho foi de $25,52 \%$ e na cobaia $17,29 \%$, diferenças estatisticamente significativas $(\mathrm{p}<0,0001)$, devido, possivelmente, à presença de fatores do tipo androgênico ou receptores celulares.

A idade também é um fator importante a ser considerado, uma vez que as características morfológicas das glândulas acessórias podem sofrer mudanças ao longo da vida do indivíduo. Na cobaia, a próstata sofre alterações morfológicas durante o envelhecimento apresentando aumento da massa estromal, fibrose e prostatite ocasional (Horsfall et al. 1994). Ao utilizar neste estudo animais adultos, sexualmente maduros, o volume glandular alcança seu tamanho máximo. Estes valores estão correlacionados diretamente com o número total de células glandulares na GBU de ambos os animais, observando-se no coelho valores significativamente maiores em relação à cobaia.

Ao se comparar as características das glândulas acessórias é importante considerar a influência do ciclo reprodutivo em cada espécie, já que pode haver alterações na morfologia glandular, características do ejaculado, fecundação e produção. Sobre isso, Chaves et al. (2012) indicaram que as glândulas anexas de roedores como a viscacha, podem experimentar alterações no seu ciclo reprodutivo anual, as quais são influenciadas por mudanças dos níveis de andrógenos que estão intimamente relacionados con as variações do fotoperíodo natural. Em animais como a chinchila, o peso da GBU apresenta os valores mais altos durante os meses de outono-inverno, coincidente com o período de maior atividade reprodutiva, tendo como consequência a hipertrofia do parênquima glandular, assim como um incremento do fluxo sanguíneo e linfático, necessários para aumentar a produção da secreção glandular (Cepeda et al. 1999). A cobaia, ao contrário, tem um ciclo estral poliéstrico não sazonal, de aproximadamente 16 dias e não depende de fatores como o fotoperíodo (Chauca 1995). No coelho, o ciclo reprodutivo não é definido. 0 macho requer uma iluminação ótima, de 8 a 16 horas/dia, razão pela qual a produção de crias diminue no outono (Cabrero 1979). Assim, o macho tem um papel importante para o êxito de uma exploração reprodutiva, uma vez que condiciona o rendimento reprodutivo das fêmeas.

\section{CONCLUSÕES}

É relevante considerar aspectos como as características morfológicas da GBU, as quais poderiam condicionar o êxito da reprodução por parte do macho. Mesmo a histologia básica da GBU sendo semelhante no coelho e na cobaia, estas glândulas apresentam diferentes valores quantitativos de peso, volume, densidade e porcentagem de tecido glandular, sendo significativamente maiores no coelho.

Em condições normais de manuseio, é relevante conhecer as características morfológicas e fisiológicas das glândulas acessórias do sistema reprodutor do macho, o que permitiria uma ótima utilização a partir de uma idade adequada, em boas condições ambientais e sazonais, possibilitando aumentar a produção e a rentabilidade na criação destes animais, os quais fazem parte da dieta de numerosas populações andinas.

\section{REFERÊNCIAS}

Ayres de Menezes D.J., Assis Neto A.C. \& Oliveira M.F. 2010. Morphology of the male agouti accessory genital glands (Dasyprocta prymnolopha Wagler, 1831). Pesq. Vet. Bras. 30(9):793-797.

Barnes G.W. 1972. The Anfigenic Nature of Male Accessory Glands of Reproduction Biol. Reprod. 6:384-421.

Cabreo E. 1979. Jornadas Cuníc. Baix Ebre. IV Reproducción-Alimentación. Boln Cunic. 6:21-29.

Cepeda R., Adaro L., Peñailillo P. \& Orostegui C. 1999. Variaciones morfológicas estacionales de las glándulas bulbouretrales de la chinchilla (Chinchilla lanier, GREY), en cautiverio. Rev. Chil. Anat. 17(1):59-66.

Chauca F.L. 1995. Producción de cuyes (Cavia porcellus) en los países andinos. Rev. Mund. Zoo. 83(2):9-19. 
Chaves E.M., Aguilera-Merlo C., Filippa V., Mohamed F., Dominguez S. \& Scardapane L. 2011. Anatomical, histological and immunohistochemical study of the reproductive system accessory glands in male viscacha ( $\mathrm{La}$ gostomus maximus maximus). Anat. Histol. Embryol. 40(1):11-20.

Chaves E.M., Aguilera-Merlo C., Cruceño A., Fogal T., Piezzi R., Scardapane L. \& Dominguez S. 2012. Seasonal morphological variations and age-related changes of the seminal vesicle of viscacha (Lagostomus maximus maximus): an ultrastructural and immunohistochemical study. Anat. Rec. 295(5):886-895.

Chughtai B., Sawas A., O'malley R.L., Naik R.R., Khan S.A. \& Pentyala S. 2005. A neglected gland: a review of Cowper's gland. Int. J. Androl. 28:74-77.

Cooper G. \& Schiller A.L. 1975. Anatomy of the Guinea Pig. Harvard University Press. Cambridge. $417 \mathrm{p}$.

Cordeiro R.S., Scarano W.R., Góes R.M. \& Taboca S.R. 2004. Tissue alterations in the Guinea pig lateral prostate following antiandrogen flutamide therapy. Biocell. 28(1):21-30.

De Barros M.A., Panattoni Martins J.F., Samoto V.Y., Oliveira V.C., Gonçalves N., Mançanares C.A., Vidane A., Carvalho A.F., Ambrósio C.E. \& Miglino M.A. 2013. Marsupial morphology of reproduction: South America opossum male model. Microsc. Res. Tech. 76(4):388-397.

Dimitrov R.S., Yonkova P.Y., Stamatova K.D. \& Yovchev D.G. 2012. Ultrasonographic features of the bulbourethral glands in the domestic rabbit (Oryctolagus cuniculus). Vet. Arhiv 82(2):193-200.

Fernandez D.S., Ferraz R.H.S., Melo A.P. F., Rodrigues R.F. \& Souza W.M. 2010. Análise histológica das glândulas uretrais da capivara (Hydrochoerus hydrochaeris). Pesq. Vet. Bras. 30(4):373-377.

Freund M. 1969. Interrelationships among the characteristics of guinea-pig semen collected by electro-ejaculation. J. Reprod. Fert. 19:393-403.

Holtz W. \& Foote R.H. 1978. The anatomy of the reproductive system in male Dutch rabbits (Oryctolagus cuniculus) with special emphasis on the accessory sex glands. J. Morphol. 158(1):1-20.

Horsfall D.J., Mayne K., Ricciardelli C., Rao M., Skinner J.M., Henderson D.W., Marshall V.R. \& Tilley W.D. 1994. Age-related changes in guinea pig prostatic stroma. Lab. Invest. 70(5):753-763.
Mann T. 1974. Secretory function of the prostate, seminal Vehicle and other male accessory organs of reproductio. J. Reprod. Fert. 37:179-188.

Martín R., Martín P. \& Gonzáles J. 2004. Atlas de Anatomía de Animales Eexóticos. Masson, Barcelona, España.

McLellan D.L., Gastón M.V., Diamond D.A., Lebowitz R.L., Mandell J., Atala A. \& Bauer S.B. 2004. Anterior urethral valves and diverticula in children: a result of ruptured Cowper's duct cyst?. BJU Int. 94(3):375-378.

Moré J. 1991. Lectin histochemistry of mucus-secreting cells in the calf bulbourethral gland. Act. Anat. 142:147-151.

Ninomiya J.G., De Coronado I.Z.P. \& Aguilar R. 1995. Fisiología Humana, Endocrinología y Metabolismo. El Manual Moderno, Santa Fé de Bogotá, p.313-314.

Orts F. 1967. Anatomía Humana. 3a ed. Científico-Médica, Barcelona. 691p.

Pedron P., Traxer O., Haab F., Farres M.T., Tligui M., Thibault P. \& Gattegno B. 1997. Glandes de Cowper: aspects anatomique, physiologique et pathologique. Progr. Urol. 7:563-569.

Scherle W. 1970. A simple method for volumetry of organs in quantitative stereology. Mikroskopie 26:57-63.

Schilling P. 1984. Temas seleccionados sobre medicina de animales de laboratorio: el cobayo. Centro Panamericano de Fiebre Aftosa, Rio de Janeiro. 81p.

Tambrallo L.J. \& Fish R.E. 2000. Laboratory Animal Medicine and Science. Series II. Guinea pigs: biology and use in research. Health Sciences Center for Educational Resources, University of Washington, Washington, DC.

Tilley W.D., Horsfall D.J., Skinner J.M., Henderson D.W. \& Marshall V.R. 1989. Effect of pubertal development on estrogen receptor levels and stromal morphology in the Guinea pig prostate. Prostate 15(2):195-210.

Vásquez B. \& del Sol M. 2001. Estudio morfológico de la glándula bulbouretral de conejo (Oryctolagus cuniculus). Rev. Chil. Anat. 19(2):221-228.

Vásquez B. \& del Sol M. 2010. Estudio morfológico de la próstata y glándulas vesiculares de Cobayo (Cavia porcellus). Int. J. Morphol. 28(4):13011307. 